\title{
PLANE CURVES IN LIE SPHERE GEOMETRY
}

\author{
TAKAYOSHI YAMAZAKI AND ATSUKo Yamada YoshiKaWA
}

\begin{abstract}
We construct Lie frames of Legendre maps into the unit tangent bundle of $S^{2}$ according to the method of moving frames, and classify them.
\end{abstract}

\section{Introduction}

Lie sphere geometry was constructed by S. Lie about 1870 , which includes Möbius geometry and Laguerre geometry. S. Sasaki and T. Suguri obtained the necessary and sufficient condition that two plane curves are Lie equivalent in 1940 ([SS]), to give an example of results; nevertheless few studies have so far been made. But there has been renewal of interest in Lie sphere geometry over the past decade. It was known that the class of Dupin hypersurfaces in $S^{n}$ is invariant under Möbius transformations. In 1985, U. Pinkall showed that this class is invariant even under Lie transformations. Since then, a large number of studies have been made on Dupin hypersurfaces in view of Lie sphere geometry by S.S. Chern, T.E. Cecil, R. Miyaoka, G. Thorbersson and so on. It may be worth while looking over the theory of plane curves in Lie sphere geometry and the contents of [SS] according to recent studies.

Let $T_{1} S^{n}$ be the unit tangent bundle of the unit sphere $S^{n}$ in the Euclidean space. An immersed hypersurface $f: M^{n-1} \rightarrow S^{n}$ with a unit normal field $\xi: M^{n-1} \rightarrow S^{n}$ naturally induces a map $\lambda=(f, \xi): M^{n-1} \rightarrow T_{1} S^{n}$. This map $\lambda$ is called the Legendre map induced by $f$ with $\xi$. The projective orthogonal group of signature $(n+1,2) P O(n+1,2)$ acts on $T_{1} S^{n}$ transitively, then $T_{1} S^{n}=$ $P O(n+1,2) / H$ for the isotropy subgroup $H$ of $P O(n+1,2)$ at a point. In this paper we are concerned with the case when $n=2$, that is Legendre maps into $T_{1} S^{2}$ induced by curves in $S^{2}$.

We apply the method of constructing Frenet frames in G. R. Jensen [J]. Let $\lambda: M \rightarrow G / H$ be a connected, smoothly embedded submanifold of a homogeneous space $G / H$. For $\lambda$, we construct a $k$-th order frame bundle $L_{k}$ which gives $k$-th order contact under the action of $G$ as follows: We begin define the zeroth order frame bundle $L_{0} \subset G$. Next we reduce $L_{0}$ to the first order frame bundle $L_{1}$ by means of considering the first order differential. Further-

Received March 31, 1995; revised December 1, 1995. 
more we reduce the frame bundle one after another in the similar way. If $\lambda$ is of constant type of any order, frames of $\lambda$ of all orders can be constructed; $L_{0} \supset L_{1} \supset L_{2} \supset \cdots \supset L_{q}=L_{q+1}$. Thus we obtain a Frenet frame $u: M \rightarrow G$ which are a lifting of $\lambda$ by taking a smooth cross section of $L_{q}$. We call $q$ the dimension of the Frenet frame. Frenet frames of a Legendre map $\lambda$ in $T_{1} S^{n}$ under the action of $P O(n+1,2)$ are called Lie frames of $\lambda$.

The summary of our main results is the following: Let $\Omega$ be the MaurerCartan form on $P O(3,2)$. If a Legendre map $\lambda: M^{1} \rightarrow T_{1} S^{2}$ is of constant type of all orders, then we get three types of Lie frames $M^{1} \rightarrow P O(3,2)$ of $\lambda$ with respect to the pullback of $\Omega$ :

Type (a): Lie frames with dimension 2 of oriented circles,

Type (b-1): Lie frames with dimension 6 of "degenerate" curves and

Type (b-2): Lie frames with dimension 8 of general curves.

Lie frames of type (b-2) agree with that obtained by S. Sasaki and T. Suguri, who examined only general curves ([SS]). This classification obtained here makes it clear how curves degenerate.

In $\S 1$, we give basic facts in Lie sphere geometry. In $\S 2$, we outline the method of constructing Lie frames, and give our main theorem. In $\S 3$, we prove the main theorem. In $\S 4$, we get some characteristics of curves in Lie equivalent classes.

This paper is based on a part of the master thesis of $\mathrm{T}$. Yamazaki ([Y]), and developed by A.Y. Yoshikawa. The authors also get some results about Lie frames of Legendre maps of surfaces ([YY]).

Thanks are due to Professor Hajime Sato for his helpful suggestions and comments in studying the problems here.

\section{Lie sphere geometry}

We give here an outline of fundamentals in Lie sphere geometry. This section is largely based on U. Pinkall $[\mathrm{P}]$ and T.E. Cecil $[\mathrm{C}]$.

\subsection{Lie spheres}

Let $S^{n}$ be the unit sphere in the Euclidean space $\boldsymbol{E}^{n+1}$, and $T_{1} S^{n}$ the unit tangent bundle of $S^{n}$; i.e.

$$
T_{1} S^{n}=\left\{(u, v) \in S^{n} \times S^{n} ; u \cdot v=0\right\},
$$

where $\cdot$ denotes the inner product of $\boldsymbol{E}^{n+1}$. An oriented hypersphere $\iota: S^{n-1}$ $\rightarrow S^{n}$ with center $p \in S^{n}$ and signed radius $\rho \in(-\pi, \pi)$ induces a mapping $(\iota, \xi): S^{n-1} \rightarrow T_{1} S^{n}$ by adding the unit normal vector field $\xi$ along $\iota$ determined by its orientation. We also call $(\iota, \xi)$ an oriented hypersphere. When $\rho=0$, that is $\iota$ shrinks to a point, we consider a mapping $(\iota, \xi)$, which is called a point sphere, by adding the inclusion $\xi: T_{1} S^{n} \rightarrow T_{1} S^{n}$. From now on, we will use the term Lie sphere to denote an oriented hypersphere or a point sphere. 
Let $\boldsymbol{R}_{2}^{n+3}=\left\{x=\left(x_{1}, \cdots, x_{n+3}\right) ; x_{i} \in \boldsymbol{R}\right\}$ be an $(n+3)$-dimensional real vector space with scalar product $\langle$,$\rangle defined by$

$$
\langle x, y\rangle=-x_{1} y_{1}+x_{2} y_{2}+\cdots+x_{n+2} y_{n+2}-x_{n+3} y_{n+3} .
$$

We denote by $\boldsymbol{P}^{n+2}$ its associated projective space, and by $\boldsymbol{Q}^{n+1}$ the quadric in $\boldsymbol{P}^{n+2}$ defined by $\langle x, x\rangle=0$. Then we can identify a Lie sphere in $T_{1} S^{n}$ with a point of $\boldsymbol{Q}^{n+1}$ under a bijective mapping from $(\iota, \xi)$ to $[\cos \rho, p, \sin \rho]$, where $p=\cos \rho \iota-\sin \rho \xi \in S^{n}$ and $\rho \in(-\pi, \pi)$.

\subsection{Lie transformations}

Let $\Lambda^{2 n-1}$ be the set of all projective lines on $\boldsymbol{Q}^{n+1}$. By Line $\{Y, Z\} \in \Lambda^{2 n-1}$ we denote the line generated by $[Y],[Z] \in \boldsymbol{Q}^{n+1}$. Then

$$
T_{1} S^{n} \cong \Lambda^{2 n-1}
$$

under a mapping from $(u, v)$ to Line $\{(1, u, 0),(0, v, 1)\}$.

A diffeomorphism $\phi: T_{1} S^{n} \rightarrow T_{1} S^{n}$ is called a Lie transformation if it carries Lie spheres to Lie spheres. For example a Möbius transformation and a parallel transformation are Lie transformations; the former takes point spheres to point spheres and the latter takes $(\iota, \xi)$ to $(\cos t \iota+\sin t \xi,-\sin t \iota+\cos t \xi)$, where $t \in[0, \pi)$. Lie transformations are generated by Möbius transformations and parallel transformations.

Denote the group of all Lie transformations by $G$. A Lie transformations $\phi$ can be regarded as a diffeomorphism $\phi: \boldsymbol{Q}^{n+1} \rightarrow \boldsymbol{Q}^{n+1}$ preserving lines on $\boldsymbol{Q}^{n+1}$, that is the restriction of a projective transformation $\Phi: \boldsymbol{P}^{n+2} \rightarrow \boldsymbol{P}^{n+2}$ preserving $\boldsymbol{Q}^{n+1}$. Thus,

$$
G \cong P O(n+1,2)=O(n+1,2) /\{ \pm 1\} .
$$

Let $o=\left(\boldsymbol{e}_{1}, \boldsymbol{e}_{n+1}\right) \in T_{1} S^{n}$ be the origin, where $\left(\boldsymbol{e}_{1}, \cdots, \boldsymbol{e}_{n+1}\right)$ is the natural basis of $\boldsymbol{E}^{n+1}$

We have chosen a basis of $\boldsymbol{R}_{2}^{n+3}$ so that it scalar product $\langle$,$\rangle is given by$ (1.1). From now on, we will change the basis of $\boldsymbol{R}_{2}^{n+3}$ so that

$$
\langle x, y\rangle={ }^{t} x S y,
$$

where

$$
S=\left(S_{\imath j}\right)=\left(\begin{array}{ccc}
0 & 0 & -I_{2} \\
0 & I_{n-1} & 0 \\
-I_{2} & 0 & 0
\end{array}\right) .
$$

Then, $O(n+1,2)=\left\{P \in G L(n+3 ; \boldsymbol{R}) ;{ }^{t} P S P=S\right\}$.

The origin $o \in T_{1} S^{n}$ is identified with Line $\{(0, \cdots, 0,1,0),(0, \cdots, 0,0,1)\} \in$ $\Lambda^{2 n-1}$. The group $G$ acts on $\Lambda^{2 n-1}$ transitively, and

$$
\Lambda^{2 n-1} \cong G / H
$$


for the isotropy subgroup $H$ of $G$ at $o$.

\subsection{Lie frames}

A Lie frame $\left(Y_{1}, \cdots, Y_{n+3}\right)$ is an ordered set of vectors in $\boldsymbol{R}_{2}^{n+3}$ satisfying the relations

$$
\left\langle Y_{\imath}, Y_{\jmath}\right\rangle=S_{\imath \jmath}
$$

for $1 \leqq i, j \leqq n+3$. The space of all Lie frames can be identified with $O(n+1,2)$.

Let $\Omega=\left(\omega_{j}^{i}\right)$ be the Maurer-Cartan form introduced by the equation

$$
d Y_{\imath}=\sum_{\jmath=1}^{n+3} \omega_{i}^{j} Y_{\jmath} .
$$

Taking the exterior derivative of (1.5), we get the Maurer-Cartan equations

$$
d \omega_{i}^{3}=\sum_{k=1}^{n+3} \omega_{i}^{k} \wedge \omega_{k}^{j}
$$

By using (1.6), we find that $\omega_{n+3}^{1} \wedge\left(d \omega_{n+3}^{1}\right)^{n-1} \neq 0$. Hence $T_{1} S^{n}$ is a contact manifold with a contact form $\omega_{n+3}^{1}$.

\subsection{Legendre submanifolds, Legendre maps and curvature spheres}

An immersed $(n-1)$-dimensional integral submanifold of the contact distribution $D$ is called a Legendre submanifold.

An immersed hypersurface $f: M^{n-1} \rightarrow S^{n}$ with a unit normal field $\xi: M^{n-1}$ $\rightarrow S^{n}$ naturally induces a Legendre submanifold $\lambda=(f, \xi): M^{n-1} \rightarrow T_{1} S^{n}$. This map $\lambda$ is called the Legendre map induced by $f$ with $\xi$. Conversely a Legendre submanifold $\lambda=(f, \xi): M^{n-1} \rightarrow T_{1} S^{n}$ naturally induces a smooth map $f: M^{n-1} \rightarrow S^{n}$, which may have singularities. The following theorem, however, shows that a Legendre submanifolds is locally transformed by a parallel transformation to be a Legendre map.

THEOREM $1.3[\mathrm{P}]$. Let $\lambda=(f, \xi): M^{n-1} \rightarrow T_{1} S^{n}$ be a Legendre submanifold. Then for each $x \in M^{n-1}$, the mapping $\cos t f+\sin t \xi: M^{n-1} \rightarrow S^{n}$ fails to be an immersion at $x$ only for at most $n-1$ values of $t \in[0, \pi)$.

Let $\lambda=$ Line $\{Y, Z\}: M^{n-1} \rightarrow \Lambda^{2 n-1}$ be a Legendre submanifold, $p \in M^{n-1}$ and $r, s \in \boldsymbol{R}$ with $(r, s) \neq(0,0)$. We call the sphere

$$
[K(p)]=[r Y(p)+s Z(p)]
$$

a curvature sphere of $\lambda$ at $p$, if there exists a non-zero vector $X$ in $T_{p} M^{n-1}$ such that

$$
r d Y(X)+s d Z(X) \in \operatorname{Span}\{Y(p), Z(p)\} .
$$

The vector $X$ is called a principal vector corresponding to $[K]$. A curvature sphere is invariant under Lie transformations. 


\section{Lie frames of Legendre maps in $T_{1} S^{2}$ under $P O(3,2)$}

We explain the method of constructing Frenet frames in the case of Lie frames of a Legendre map $\lambda$ from a 1-dimensional manifold $M$ to $T_{1} S^{2}$.

First of all we construct a zeroth order frame field along $\lambda$. The isotropy subgroup $H$ of $G$ at the origin $o$ is given by

$$
\begin{gathered}
H=\left\{\left(\begin{array}{ccc}
A & 0 & 0 \\
0 & 1 & 0 \\
0 & 0 & { }^{t} A^{-1}
\end{array}\right) \exp \left(\begin{array}{ccc}
0 & 0 & 0 \\
B & 0 & 0 \\
0 & { }^{t} B & 0
\end{array}\right) \exp \left(\begin{array}{ccc}
0 & 0 & 0 \\
0 & 0 & 0 \\
C & 0 & 0
\end{array}\right) ;\right. \\
\left.A \in G L(2 ; \boldsymbol{R}), B \in M_{1,2}(\boldsymbol{R}), C \in \mathfrak{D}(2)\right\} .
\end{gathered}
$$

From now on we denote the matrices $\left(\begin{array}{ccc}A & 0 & 0 \\ 0 & 1 & 0 \\ 0 & 0 & { }^{t} A^{-1}\end{array}\right), \exp \left(\begin{array}{ccc}0 & 0 & 0 \\ B & 0 & 0 \\ 0 & { }^{t} B & 0\end{array}\right)$ and $\exp \left(\begin{array}{lll}0 & 0 & 0 \\ 0 & 0 & 0 \\ C & 0 & 0\end{array}\right)$ by $\tilde{A}, \tilde{B}$ and $\tilde{C}$, and denote a matrix $h$ in $H$ by $h=\tilde{A} \tilde{B} \tilde{C}$. The Lie algebra $g$ of $G$ is given by

$$
\begin{aligned}
\mathfrak{g} & =\left\{X \in \mathfrak{g l}(n+3 ; \boldsymbol{R}) ;{ }^{t} X S+S X=0\right\} \\
& =\left\{\left(\begin{array}{ccc}
\alpha & \delta & \zeta \\
\beta & 0 & { }^{t} \delta \\
\gamma & { }^{t} \beta & -{ }^{t} \alpha
\end{array}\right) ; \alpha \in M_{2,2}(\boldsymbol{R}), \beta,{ }^{t} \delta \in M_{1,2}(\boldsymbol{R}), \gamma, \zeta \in \mathfrak{D}(2)\right\},
\end{aligned}
$$

and the Lie algebra $\mathfrak{h}$ of $H$ is given by

$$
\mathfrak{h}=\left\{\left(\begin{array}{ccc}
\alpha & 0 & 0 \\
\beta & 0 & 0 \\
\gamma & { }^{t} \beta & -{ }^{t} \alpha
\end{array}\right) ; \alpha \in M_{2,2}(\boldsymbol{R}), \beta \in M_{1,2}(\boldsymbol{R}), \gamma \in \mathfrak{v}(2)\right\} \text {. }
$$

We take a vector subspace $\mathfrak{m}$ of $\mathfrak{g}$ complementary to $\mathfrak{h}$, and choose the following $e_{1}, e_{2}, e_{3}$ for a basis of $\mathrm{m}$ :

$$
\begin{aligned}
& e_{1}=\left(\begin{array}{cc|c|cc}
0 & 0 & 0 & 0 & 1 \\
0 & 0 & 0 & -1 & 0 \\
\hline 0 & 0 & 0 & 0 & 0 \\
\hline 0 & 0 & 0 & 0 & 0 \\
0 & 0 & 0 & 0 & 0
\end{array}\right), \quad e_{2}=\left(\begin{array}{ll|l|ll}
0 & 0 & 1 & 0 & 0 \\
0 & 0 & 0 & 0 & 0 \\
\hline 0 & 0 & 0 & 1 & 0 \\
\hline 0 & 0 & 0 & 0 & 0 \\
0 & 0 & 0 & 0 & 0
\end{array}\right) \\
& e_{3}=\left(\begin{array}{ll|l|ll}
0 & 0 & 0 & 0 & 0 \\
0 & 0 & 1 & 0 & 0 \\
\hline 0 & 0 & 0 & 0 & 1 \\
\hline 0 & 0 & 0 & 0 & 0 \\
0 & 0 & 0 & 0 & 0
\end{array}\right) .
\end{aligned}
$$


Let $\rho_{0}: H \rightarrow G L(3, \boldsymbol{R})$ be the isotropy representation given by the adjoint action of $H$ on $\mathfrak{m}$ with respect to this basis. We denote $A$ by $\left(\begin{array}{ll}a_{11} & a_{12} \\ a_{21} & a_{22}\end{array}\right)$, then for $h \in H$,

$$
\rho_{0}(h)=\left(\begin{array}{ccc}
\operatorname{det} A & 0 & 0 \\
* & a_{11} & a_{12} \\
* & a_{21} & a_{22}
\end{array}\right) .
$$

A zeroth order frame at $p \in M$ is an element $g \in G$ such that $\pi(g)=\lambda(p)$ (where $\pi$ is the natural projection $G \rightarrow G / H)$. Let $L_{0}$ denote the set of all zeroth order frames on $M$. A zeroth order frame field $u$ along $\lambda$ is a smooth cross section of $L_{0} \rightarrow M$.

In the next step, we construct a first order frame field along $\lambda$. Let $\Omega=\left(\omega_{j}^{i}\right)$ be the Maurer-Cartan form on $G$. With respect to the decomposition $\mathfrak{g}=\mathfrak{h}+\mathfrak{m}$, we decompose $\Omega$ into $\Omega_{0}+\Theta_{0}$, so we get

$$
\Theta_{0}=\omega_{5}^{1} e_{1}+\omega_{3}^{1} e_{2}+\omega_{3}^{2} e_{3} .
$$

We see that $u^{*} \omega_{5}^{1}=0$, because $\lambda$ is a Legendre submanifold and $\omega_{5}^{1}$ is the contact form. We choose a line element $\phi$ of $M$. and set $u^{*} \omega_{3}^{1}=x_{2} \phi, u^{*} \omega_{3}^{2}=x_{3} \phi$ for some smooth functions $x_{2}, x_{3}$ on $M$. Let $u$ be a zeroth order frame field along $\lambda$. We denote by $R_{u}$ the right action of $u \in G$ on $G / H$. We define a smooth map $\lambda_{0}$ from $L_{0}$ to the Grassmann manifold $G_{3,1}$ so that $\lambda_{0}(u)=R_{u^{*}}^{-1} \lambda_{*}\left(T_{p} M\right)$ where $\lambda(p)=\pi(u)$ by identifying $\mathfrak{m} \cong T_{o}(G / H)$ with $\boldsymbol{R}^{3}$ with respect to the basis $e_{1}, e_{2}, e_{3}$ of $\mathrm{m}$, then.

$$
\lambda_{0}(u)={ }^{t}\left[\begin{array}{lll}
0 & x_{2}(p) & \left.x_{3}(p)\right] \in G_{3,1} .
\end{array}\right.
$$

(For further details, see [J].) Let $V$ be a neighborhood of $u$ in $L_{0}$. We denote a point ${ }^{t}\left[\begin{array}{lll}0 & x_{2} & x_{3}\end{array}\right] \in G_{3,1}$ by $[\boldsymbol{x}]$. We consider the orbit $\mathcal{O}_{1}$ on $\lambda_{0}(V)$ under the action of $\left(H, \rho_{0}\right)$, that is $\mathcal{O}_{1}=\bigcup_{[x] \in \lambda_{0}(V)}\left\{\rho_{0}(h)[x] \mid h \in H\right\}$. The action of $\left(H, \rho_{0}\right)$ is as follows :

$$
\rho_{0}(\tilde{A} \tilde{B} \tilde{C})[\boldsymbol{x}]={ }^{t}\left[\begin{array}{lll}
0 & a_{11} x_{2}+a_{12} x_{3} & a_{21} x_{2}+a_{22} x_{3}
\end{array}\right] .
$$

There exists a smooth map $A_{V}: V \rightarrow G L(2, \boldsymbol{R})$ such that $A_{V}(v)\left[\begin{array}{l}x_{2}(q) \\ x_{3}(q)\end{array}\right]=\left[\begin{array}{l}1 \\ 0\end{array}\right]$ for $v \in V$ and $\pi(v)=\lambda(q)$. Thus we can take a local cross section $s$ so that $s\left(\left[\begin{array}{c}0 \\ x_{2}(q) \\ x_{3}(q)\end{array}\right]\right)=\rho_{0}\left(\widetilde{A_{V}(v)} \tilde{O} \tilde{O}\right)\left[\begin{array}{c}0 \\ x_{2}(q) \\ x_{3}(q)\end{array}\right]=\left[\begin{array}{l}0 \\ 1 \\ 0\end{array}\right]$. Let $W_{1}=s\left(\lambda_{0}(V)\right) \subset G_{3,1}$, that is

$$
W_{1}=\left\{{ }^{t}\left[\begin{array}{lll}
0 & 1 & 0
\end{array}\right]\right\} \text {. }
$$

We say that $\lambda$ has the type of $W_{1}$ if there exists a zeroth order frame field $u$ such that $\lambda_{0}(u) \subset W_{1}$. If $\lambda$ has the type of $W_{1}$, we let $L_{1}=\lambda_{0}^{-1}\left(W_{1}\right)$ and call $L_{1}$ the set of first order frames on $\lambda$ (with respect to $W_{1}$ ). 
The isotropy subgroup $H_{1}$ of $H$ at a point of $W_{1}$ is given by

$$
H_{1}=\left\{\tilde{A} \tilde{B} \tilde{C} ; A=\left(\begin{array}{cc}
a_{11} & a_{12} \\
0 & a_{22}
\end{array}\right) \in G L(2, \boldsymbol{R}), B \in M_{1,2}(\boldsymbol{R}), C \in \mathfrak{v}(2)\right\} .
$$

We say that $\lambda$ is of constant type at zeroth order if there exists an neighborhood $U$ of $p$ on $M$ and a local cross section $W_{1}$ such that $\left.\lambda\right|_{U}$ is the type of $W_{1}$ and the isotropy subgroup of $H$ at any point of $W_{1}$ is equal to $H_{1}$. In this case $\lambda$ is always of constant type of zeroth order. We reach the conclusion that the first order frame field $u$ satisfies

$$
u^{*}\left(\Theta_{0}\right)=\phi e_{2},
$$

and that any two Legendre maps in $T_{1} S^{2}$ have at least first order $G$-contact.

In the third place, we construct a second order frame field along $\lambda$. The Lie algebra $\mathfrak{h}_{1}$ of $H_{1}$ is given by

$$
\mathfrak{h}_{1}=\left\{\left(\begin{array}{ccc}
\alpha & 0 & 0 \\
\beta & 0 & 0 \\
\gamma & { }^{t} \beta & -{ }^{t} \alpha
\end{array}\right) ; \alpha=\left(\begin{array}{cc}
\alpha_{11} & \alpha_{12} \\
0 & \alpha_{22}
\end{array}\right), \beta \in M_{1,2}(\boldsymbol{R}), \gamma \in \mathfrak{D}(2)\right\} .
$$

We decompose $\mathfrak{h}=\mathfrak{h}_{1}+\mathfrak{m}_{1}$, and for a basis of $\mathfrak{m}_{1}$ we take the following $e_{4}$.

$$
e_{4}=\left(\begin{array}{cc|c|cc}
0 & 0 & 0 & 0 & 0 \\
1 & 0 & 0 & 0 & 0 \\
\hline 0 & 0 & 0 & 0 & 0 \\
\hline 0 & 0 & 0 & 0 & -1 \\
0 & 0 & 0 & 0 & 0
\end{array}\right) .
$$

The isotropy representation $\rho_{1}: H_{1} \rightarrow G L(4, \boldsymbol{R})$ given by the adjoint action of $H_{1}$ on $\mathfrak{m}+\mathfrak{m}_{1}$ with respect to the basis $e_{1}, \cdots, e_{4}$ is

$$
\rho_{1}(h)=\left(\begin{array}{cccc}
* & 0 & * & 0 \\
* & a_{11} & * & 0 \\
* & 0 & * & 0 \\
* & 0 & * & \frac{a_{22}}{a_{11}}
\end{array}\right),
$$

where $h=\tilde{A} \tilde{B} \tilde{C} \in H_{1}$, and $A=\left(\begin{array}{cc}a_{11} & a_{12} \\ 0 & a_{22}\end{array}\right) \in G L(2, \boldsymbol{R})$. Let $u$ be a first order frame field along $\lambda$. With respect to the decomposition $\mathfrak{h}=\mathfrak{h}_{1}+\mathfrak{m}_{1}$, we decompose $\Omega_{0}$ into $\Omega_{1}+\Theta_{1}$, then

$$
\Theta_{1}=\omega_{1}^{2} e^{4} .
$$


Set $u^{*} \omega_{1}^{2}=x_{4} \phi$ for a smooth function $x_{4}$ on $M$. We denote by $R_{u}^{(1)}$ the right action of $u \in G$ on $G / H_{1}$. Let $\lambda^{(1)}: M \rightarrow G / H_{1}$ be given by $\lambda^{(1)}(p)=[u]$ where $\lambda(p)=\pi(u)$. We define a smooth map $\lambda_{1}$ from $L_{1}$ to $G_{4,1}$ so that $\lambda_{1}(u)+$ $R_{u^{-1 *}(1)}^{(1)} \lambda_{*}^{(1)}\left(T_{p} M\right)$ by identifying $\mathfrak{m}+\mathfrak{m}_{1} \cong T_{[o]}\left(G / H_{1}\right)$, with $\boldsymbol{R}^{4}$ with respect to $e_{1}, \cdots, e_{4}$, then

$$
\lambda_{1}(u)={ }^{t}\left[\begin{array}{llll}
0 & 1 & 0 & \left.x_{4}(p)\right] \in G_{4,1} .
\end{array}\right.
$$

We consider the orbits on $\lambda_{1}(V)$ under the action of $\left(H_{1}, \rho_{1}\right)$. We denote a point ${ }^{t}\left[\begin{array}{llll}0 & 1 & 0 & x_{4}\end{array}\right]$ in $G_{4,1}$ by $[\boldsymbol{x}]$. The action of $\left(H_{1}, \rho_{1}\right)$ is as follows :

$$
\rho_{1}(\tilde{A} \tilde{B} \tilde{C})[\boldsymbol{x}]={ }^{t}\left[\begin{array}{llll}
0 & a_{11} & 0 & \frac{a_{22}}{a_{11}} x_{4}
\end{array}\right]={ }^{t}\left[\begin{array}{llll}
0 & 1 & 0 & \frac{a_{22}}{\left(a_{11}\right)^{2}} x_{4}
\end{array}\right] \text {. }
$$

Let $V$ be a neighborhood of $u$ in $L_{1}$, and let

$$
\begin{aligned}
& G_{4,1}^{a}=\left\{t\left[\begin{array}{llll}
0 & 1 & 0 & 0
\end{array}\right]\right\} \subset G_{4,1}, \\
& G_{4,1}^{b}=\left\{t\left[\begin{array}{llll}
0 & 1 & 0 & x
\end{array}\right] ; x \neq 0\right\} \subset G_{4,1} .
\end{aligned}
$$

Suppose that $\lambda_{1}(V) \subset G_{4,1}^{a}$, and denote the orbit on $\lambda(V)$ by $\mathcal{O}_{2}^{a}$, that is $\mathcal{O}_{2}^{a}=$

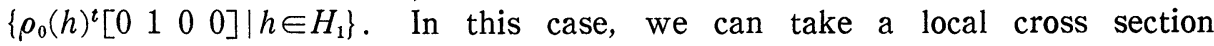
$s: \lambda_{1}(V) \rightarrow \mathcal{O}_{2}^{a}$ so that $s\left(\left[\begin{array}{l}0 \\ 1 \\ 0 \\ 0\end{array}\right]\right)=\left[\begin{array}{l}0 \\ 1 \\ 0 \\ 0\end{array}\right]$, and hence

$$
W_{2}^{a}=s\left(\lambda_{1}(V)\right)=\left\{t\left[\begin{array}{llll}
0 & 1 & 0 & 0
\end{array}\right]\right\} .
$$

We say that $\lambda$ has the type of $W_{2}^{a}$ if there exists a first order frame field $u$ such that $\lambda_{1}(u) \subset W_{2}^{a}$. If $\lambda$ has the type of $W_{2}^{a}$, we let $L_{2}^{a}=\lambda_{0}^{-1}\left(W_{2}^{a}\right)$ and call $L_{2}^{a}$ the set of second order frames on $\lambda$ with respect to $W_{2}^{a}$.

Suppose that $\lambda_{1}(V) \subset G_{4,1}^{b}$, and denote the orbit on $\lambda(V)$ by $\mathcal{O}_{2}^{b}$, that is $\mathcal{O}_{2}^{b}=\bigcup_{[x] \in G_{4,1}^{b}}\left\{\rho_{0}(h)[\boldsymbol{x}] \mid h \in H_{1}\right\}$. In this case, we can take a local cross section $s: \lambda_{1}(V) \rightarrow \Theta_{2}^{b}$ so that $s\left(\left[\begin{array}{l}0 \\ 1 \\ 0 \\ x\end{array}\right]\right)=\left[\begin{array}{l}0 \\ 1 \\ 0 \\ 1\end{array}\right]$, and hence

$$
W_{2}^{b}=s\left(\lambda_{1}(V)\right)=\left\{t^{t}\left[\begin{array}{llll}
0 & 1 & 0 & 1
\end{array}\right]\right\} .
$$

We say that $\lambda$ has the type of $W_{2}^{b}$ if there exists a first order frame field $u$ such that $\lambda_{1}(u) \subset W_{2}^{b}$. If $\lambda$ has the type of $W_{2}^{b}$, we let $L_{2}^{b}=\lambda_{0}^{-1}\left(W_{2}^{b}\right)$ and call $L_{2}^{b}$ the set of second order frames on $\lambda$ with respect to $W_{2}^{b}$.

The isotropy subgroup $H_{2}^{a}$ of $H_{1}$ at a point of $W_{2}^{a}$ is equal to $H_{1}$, and the isotropy subgroup $H_{1}^{b}$ of $H_{1}$ at a point of $W_{2}^{b}$ is given by

$$
H_{2}^{b}=\left\{\tilde{A} \tilde{B} \tilde{C} ; A=\left\{\begin{array}{cc}
a_{1} & a_{2} \\
0 & \left(a_{1}\right)^{2}
\end{array}\right) \in G L(2, \boldsymbol{R}), B=\left(\begin{array}{ll}
b_{1} & b_{2}
\end{array}\right), C \in \mathfrak{0}(2)\right\} .
$$


We say that $\lambda$ is of constant type (a) or type (b) at first order if there exists an neighborhood $U$ of $p$ on $M$ such that $\left.\lambda\right|_{U}$ is the type of $W_{1}^{a}$ or $W_{1}^{b}$ respectively.

Suppose that $\lambda$ is of constant type (a) at first order. Then we get a second order frame field $u: U \rightarrow G / H_{1}$ along $\lambda: U \rightarrow G / H$. Adding an extra step, we lift $u$ from $G / H_{1}$ to $G$. We choose here a lifting $\tilde{u}$ of $u$ such that $\tilde{u}^{*} \Omega_{1}=0$, then we obtain the Lie frame $\tilde{u}: U \rightarrow G$ of type (a) with dimension 2:

$$
u^{*} \Omega=X_{a} \phi \text {, where } X_{a}=\left(\begin{array}{cc|c|cc}
0 & 0 & 1 & 0 & 0 \\
0 & 0 & 0 & 0 & 0 \\
\hline 0 & 0 & 0 & 1 & 0 \\
\hline 0 & 0 & 0 & 0 & 0 \\
0 & 0 & 0 & 0 & 0
\end{array}\right) .
$$

Suppose that $\lambda$ is of constant type (b) at first order. Furthermore we reduce the frames one after another in the similar way. Suppose that there is an open set $U$ such that $\left.\lambda\right|_{U}$ is of constant type of any order, then we can construct frames of all orders : $L_{0} \supset L_{1} \supset L_{2} \supset \cdots \supset L_{q}=L_{q+1}$. Thus we obtain a Lie frame $U \rightarrow G$ of type (b) which is a lifting of $\lambda$ by means of taking a smooth cross section of $L_{q}$. We will describe the further process of constructing higher order frames in detail in $\S 3$. In conclusion we get the following main theorem:

THEOREM 2.1. Let $\lambda: M^{1} \rightarrow T_{1} S^{2}$ be a Legendre map which is induced by an embedded curve $f: M^{1} \rightarrow S^{2}$ with field of unit normals $\xi: M^{1} \rightarrow S^{2}$. Let $\Omega$ be the Maurer-Cartan form on $P O(3,2)$ and $d s$ a line element on $M^{1}$. If there exists an open set $U \subset M^{1}$ such that $\left.\lambda\right|_{U}$ is of constant type of any order, then we can construct one of the following three types of Lie frames $u: U \rightarrow P O(3,2)$ of $\left.\lambda\right|_{U}$ : Type (a):

$$
u^{*} \Omega=X_{a} d s, \text { where } X_{a}=\left(\begin{array}{cc|c|cc}
0 & 0 & 1 & 0 & 0 \\
0 & 0 & 0 & 0 & 0 \\
\hline 0 & 0 & 0 & 1 & 0 \\
\hline 0 & 0 & 0 & 0 & 0 \\
0 & 0 & 0 & 0 & 0
\end{array}\right) .
$$

Type (b-1):

$$
u^{*} \Omega=X_{b}^{1} d s, \text { where } X_{b}^{1}=\left(\begin{array}{cc|c|cc}
0 & 0 & 1 & 0 & 0 \\
1 & 0 & 0 & 0 & 0 \\
\hline 0 & 0 & 0 & 1 & 0 \\
\hline 0 & 0 & 0 & 0 & -1 \\
0 & 0 & 0 & 0 & 0
\end{array}\right) \text {. }
$$


Type (b-2):

$$
u^{*} \Omega=X_{b}^{2} d s \text {, where } X_{b}^{2}=\left(\begin{array}{cc|c|cc}
0 & k & 1 & 0 & 0 \\
1 & 0 & 0 & 0 & 0 \\
\hline \frac{3}{2} k & 0 & 0 & 1 & 0 \\
\hline 0 & -1 & \frac{3}{2} k & 0 & -1 \\
1 & 0 & 0 & -k & 0
\end{array}\right) \text {, }
$$

where $k$ is the seventh order invariant of $\lambda$.

To put the condition of type (b-2) in another way, we can take a Lie frame $\left(Y_{1}, Y_{2}, Y_{3}, Y_{4}, Y_{5}\right)$ that satisfies the following Frenet's formula:

$$
\begin{aligned}
& \frac{d Y_{1}}{d s}=Y_{2}+\frac{3}{2} k Y_{3}+Y_{5} \\
& \frac{d Y_{2}}{d s}=k Y_{1}-Y_{4} \\
& \frac{d Y_{3}}{d s}=Y_{1}+\frac{3}{2} k Y_{4} \\
& \frac{d Y_{4}}{d s}=Y_{3}-k Y_{5} \\
& \frac{d Y_{5}}{d s}=-Y_{4} .
\end{aligned}
$$

This formula agrees with that obtained by S. Sasaki and T. Suguri ([SS]).

\section{Proof of Theorem 2.1}

We have already explained how to construct second order frames in the preceding section. We continue constructing higher order frames of type (b).

Construction of third order frames of type (b).

The Lie algebra $\mathfrak{h}_{2}$ of $H_{2}^{b}$ is given by

$$
\mathfrak{h}_{2}=\left\{\left(\begin{array}{ccc}
\alpha & 0 & 0 \\
\beta & 0 & 0 \\
\gamma & { }^{t} \beta & -{ }^{t} \alpha
\end{array}\right) ; \alpha=\left(\begin{array}{cc}
\alpha_{1} & \alpha_{2} \\
0 & 2 \alpha_{1}
\end{array}\right), \beta \in M_{1,2}(\boldsymbol{R}), \gamma \in \mathfrak{p}(2)\right\}
$$

We decompose $\mathfrak{h}_{1}=\mathfrak{h}_{2}+\mathfrak{m}_{2}$, and for a basis of $\mathfrak{m}_{2}$ we take 


$$
e_{5}=\left(\begin{array}{cc|c|cc}
0 & 0 & 0 & 0 & 0 \\
0 & 1 & 0 & 0 & 0 \\
\hline 0 & 0 & 0 & 0 & 0 \\
\hline 0 & 0 & 0 & 0 & 0 \\
0 & 0 & 0 & 0 & -1
\end{array}\right) .
$$

The representation $\rho_{2}: H_{2}^{b} \rightarrow G L(5, \boldsymbol{R})$ given by the adjoint action of $H_{2}^{b}$ on $\mathfrak{m}+\mathfrak{m}_{1}+\mathfrak{m}_{2}$ with respect to the basis $e_{1}, \cdots, e_{5}$ is

$$
\rho_{2}(P)=\left(\begin{array}{ccccc}
* & 0 & * & 0 & 0 \\
* & a_{1} & * & 0 & 0 \\
* & 0 & * & 0 & 0 \\
* & 0 & * & a_{1} & 0 \\
* & 2 b_{1} & * & -\frac{3 a_{2}}{a_{1}} & 1
\end{array}\right),
$$

where $P \in H_{2}^{b}$. With respect to $\mathfrak{h}_{1}=\mathfrak{h}_{2}+\mathfrak{m}_{2}$, we have $\Omega_{1}=\Omega_{2}+\Theta_{2}$, where

$$
\Theta_{2}=\left(\omega_{2}^{2}-2 \omega_{1}^{1}\right) e_{5} \text {. }
$$

We consider orbits and local sections $W_{3}$ of the action of $\left(H_{2}^{b}, \rho_{2}\right)$ on $G_{5,1}$. We can take

$$
\left.W_{3}=\left\{\begin{array}{lllll}
0 & 1 & 0 & 1 & 0
\end{array}\right]\right\} \text {. }
$$

Construction of fourth order frames of type (b).

The isotropy subgroup $H_{3}$ of $H_{2}^{b}$ at a point of $W_{3}$ is given by

$$
H_{3}=\left\{\tilde{A} \tilde{B} \tilde{C} ; A=\left(\begin{array}{cc}
a & \frac{2}{3} a b_{1} \\
0 & (a)^{2}
\end{array}\right) \in G L(2, \boldsymbol{R}), B=\left(\begin{array}{ll}
b_{1} & b_{2}
\end{array}\right), C \in \mathfrak{D}(2)\right\} .
$$

The Lie algebra $\mathfrak{h}_{3}$ of $H_{3}$ is given by

$$
\mathfrak{h}_{3}=\left\{\left(\begin{array}{ccc}
\alpha & 0 & 0 \\
\beta & 0 & 0 \\
\gamma & { }^{t} \beta & -{ }^{t} \alpha
\end{array}\right) ; \alpha=\left(\begin{array}{cc}
\alpha_{1} & \frac{2}{3} \beta_{1} \\
0 & 2 \alpha_{1}
\end{array}\right), \beta=\left(\beta_{1} \beta_{2}\right), \gamma \in \mathfrak{D}(2)\right\} \text {. }
$$

We decompose $\mathfrak{h}_{2}=\mathfrak{h}_{3}+\mathfrak{m}_{3}$, and for a basis of $\mathfrak{m}_{3}$ we take

$$
e_{6}=\left(\begin{array}{cc|c|rc}
0 & 1 & 0 & 0 & 0 \\
0 & 0 & 0 & 0 & 0 \\
\hline 0 & 0 & 0 & 0 & 0 \\
\hline 0 & 0 & 0 & 0 & 0 \\
0 & 0 & 0 & -1 & 0
\end{array}\right) .
$$


The representation $\rho_{3}: H_{3} \rightarrow G L(6, \boldsymbol{R})$ given by the adjoint action of $H_{3}$ on $\mathfrak{m}+\mathfrak{m}_{1}+\mathfrak{m}_{2}+\mathfrak{m}_{3}$ with respect to the basis $e_{1}, \cdots, e_{6}$ is

$$
\rho_{3}(P)=\left(\begin{array}{cccccc}
* & 0 & * & 0 & * & 0 \\
* & a & * & 0 & * & 0 \\
* & 0 & * & 0 & * & 0 \\
* & 0 & * & a & * & 0 \\
* & 2 b_{1} & * & -2 b_{1} & * & 0 \\
* \frac{\left(b_{1}\right)^{2}-b_{2}}{a} * \frac{-4\left(b_{1}\right)^{2}-6 b_{2}}{9 a} & * & \frac{1}{a}
\end{array}\right) \text {, }
$$

where $P \in H_{3}$. With respect to $\mathfrak{h}_{2}=\mathfrak{h}_{3}+\mathfrak{m}_{3}$, we have $\Omega_{2}=\Omega_{3}+\Theta_{3}$, where

$$
\Theta_{3}=\left(\omega_{2}^{1}-\frac{2}{3} \omega_{1}^{3}\right) e_{6} .
$$

We consider orbits and local sections $W_{4}$ of the action of $\left(H_{3}, \rho_{3}\right)$ on $G_{6,1}$. We can take

$$
W_{4}=\left\{t\left[\begin{array}{llllll}
0 & 1 & 0 & 1 & 0 & 0
\end{array}\right]\right\} .
$$

Construction of fifth order frames of type (b).

The isotropy subgroup $H_{4}$ of $H_{3}$ at a point of $W_{4}$ is given by

$$
H_{4}=\left\{\tilde{A} \tilde{B} \tilde{C} ; A=\left(\begin{array}{cc}
a & \frac{2}{3} a b \\
0 & (a)^{2}
\end{array}\right) \in G L(2, \boldsymbol{R}), B=\left(b \frac{1}{3}(b)^{2}\right), C=\left(\begin{array}{cc}
0 & c \\
-c & 0
\end{array}\right)\right\} \text {. }
$$

The Lie algebra $\mathfrak{h}_{4}$ of $H_{4}$ is given by

$$
\mathfrak{h}_{4}=\left\{\left(\begin{array}{ccc}
\alpha & 0 & 0 \\
\beta & 0 & 0 \\
\gamma & { }^{t} \beta & -{ }^{t} \alpha
\end{array}\right) ; \alpha=\left(\begin{array}{cc}
\alpha_{1} & \frac{2}{3} \beta_{1} \\
0 & 2 \alpha_{1}
\end{array}\right), \beta=\left(\beta_{1} 0\right), \gamma \in \mathfrak{D}(2)\right\} .
$$

We decompose $\mathfrak{h}_{3}=\mathfrak{h}_{4}+\mathfrak{m}_{4}$, and for a basis of $\mathfrak{m}_{4}$ we take

$$
e_{7}=\left(\begin{array}{cc|c|cc}
0 & 0 & 0 & 0 & 0 \\
0 & 0 & 0 & 0 & 0 \\
\hline 0 & 1 & 0 & 0 & 0 \\
\hline 0 & 0 & 0 & 0 & 0 \\
0 & 0 & 1 & 0 & 0
\end{array}\right) .
$$

The representation $\rho_{4}: H_{4} \rightarrow G L(7, \boldsymbol{R})$ given by the adjoint action of $H_{4}$ on $\mathfrak{m}+\mathfrak{m}_{1}+\cdots+\mathfrak{m}_{4}$ with respect to the basis $e_{1}, \cdots, e_{7}$ is 


$$
\rho_{4}(P)=\left(\begin{array}{ccccccc}
* & 0 & * & 0 & * & * & 0 \\
* & a & * & 0 & * & * & 0 \\
* & 0 & * & 0 & * & * & 0 \\
* & 0 & * & a & * & * & 0 \\
* & 2 b & * & -2 b & * & * & 0 \\
* & \frac{2(b)^{2}}{3 a} & * & -\frac{2(b)^{2}}{3 a} & * & * & 0 \\
* \frac{(b)^{3}-6 c}{6(a)^{2}} & * & -\frac{2(b)^{3}}{9(a)^{2}} & * & * & \frac{1}{(a)^{2}}
\end{array}\right),
$$

where $P \in H_{4}$. With respect to $\mathfrak{h}_{3}=\mathfrak{h}_{4}+\mathfrak{m}_{4}$, we have $\Omega_{3}=\Omega_{4}+\Theta_{4}$, where

$$
\Theta_{4}=\omega_{2}^{3} e_{7} .
$$

We consider orbits and local sections $W_{5}$ of the action of $\left(H_{4}, \rho_{4}\right)$ on $G_{7,1}$. We can take

$$
\left.W_{5}=\left\{\begin{array}{lllllll}
t_{0} & 1 & 0 & 1 & 0 & 0 & 0
\end{array}\right]\right\} .
$$

Construction of sixth order frames of type (b).

The isotropy subgroup $H_{5}$ of $H_{4}$ at a point of $W_{5}$ is given by

$$
H_{5}=\left\{\tilde{A} \tilde{B} \tilde{C} ; A=\left(\begin{array}{cc}
a & \frac{2}{3} a b \\
0 & (a)^{2}
\end{array}\right) \in G L(2, \boldsymbol{R}), B=\left(b \frac{1}{3}(b)^{2}\right), C=\left(\begin{array}{cc}
0 & -\frac{(b)^{3}}{18} \\
\frac{(b)^{3}}{18} & 0
\end{array}\right)\right\} \text {. }
$$

The Lie algebra $\mathfrak{h}_{5}$ of $H_{5}$ is given by

$$
\mathfrak{h}_{5}=\left\{\left(\begin{array}{ccc}
\alpha & 0 & 0 \\
\beta & 0 & 0 \\
0 & { }^{t} \beta & -{ }^{t} \alpha
\end{array}\right) ; \alpha=\left(\begin{array}{cc}
\alpha_{1} & \frac{2}{3} \beta_{1} \\
0 & 2 \alpha_{1}
\end{array}\right), \beta=\left(\begin{array}{ll}
\beta_{1} & 0
\end{array}\right)\right\} .
$$

We decompose $\mathfrak{h}_{4}=\mathfrak{h}_{5}+\mathfrak{m}_{5}$, and for a basis of $\mathfrak{m}_{5}$ we take

$$
e_{8}=\left(\begin{array}{rr|r|rr}
0 & 0 & 0 & 0 & 0 \\
0 & 0 & 0 & 0 & 0 \\
\hline 0 & 0 & 0 & 0 & 0 \\
\hline 0 & 1 & 0 & 0 & 0 \\
-1 & 0 & 0 & 0 & 0
\end{array}\right) .
$$

The representation $\rho_{5}: H_{5} \rightarrow G L(8, \boldsymbol{R})$ given by the adjoint action of $H_{5}$ on $\mathfrak{m}+\mathfrak{m}_{1}+\cdots+\mathfrak{m}_{5}$ with respect to the basis $e_{1}, \cdots, e_{8}$ is 


$$
\rho_{5}(P)=\left(\begin{array}{cccccccc}
* & 0 & * & 0 & * & * & * & 0 \\
* & a & * & 0 & * & * & * & 0 \\
* & 0 & * & 0 & * & * & * & 0 \\
* & 0 & * & a & * & * & * & 0 \\
* & 2 b & * & -2 b & * & * & * & 0 \\
* & \frac{2(b)^{2}}{3 a} & * & -\frac{2(b)^{2}}{3 a} & * & * & * & 0 \\
* & \frac{2(b)^{3}}{9(a)^{2}} & * & -\frac{2(b)^{3}}{9(a)^{2}} & * & * & * & 0 \\
* \frac{(b)^{4}}{18(a)^{3}} & * & -\frac{(b)^{4}}{18(a)^{3}} & * & * & * & \frac{1}{(a)^{3}}
\end{array}\right),
$$

where $P \in H_{5}$. With respect to $\mathfrak{h}_{4}=\mathfrak{h}_{5}+\mathfrak{m}_{5}$, we have $\Omega_{4}=\Omega_{5}+\Theta_{5}$, where

$$
\Theta_{5}=\omega_{2}^{4} e_{8} \text {. }
$$

We consider orbits and local sections $W_{6}$ of the action of $\left(H_{5}, \rho_{5}\right)$ on $G_{8,1}$. We can take the following two types of $W_{6}$ :

$$
W_{6}^{1}=\left\{{ }^{t}\left[\begin{array}{llllllll}
0 & 1 & 0 & 1 & 0 & 0 & 0 & 0
\end{array}\right], \quad W_{6}^{2}=\left\{{ }^{t}\left[\begin{array}{llllllll}
0 & 1 & 0 & 1 & 0 & 0 & 0 & -1
\end{array}\right]\right\} .\right.
$$

The isotropy subgroup $H_{6}^{1}$ of $H_{5}$ at a point of $W_{6}^{1}$ is equal to $H_{5}$. Suppose that there is a small open set $U$ in $M$ such that $\left.\lambda\right|_{U}$ is of constant type at first order with respect to $W_{6}^{1}$; We say that $\lambda$ is of type (b-1) in $U$. In this way we get a sixth order frame field $u: U \rightarrow G / H_{5}$ along $\lambda: U \rightarrow G / H$. Adding an extra step, we lift $u$ from $G / H_{5}$ to $G$. We choose here a lifting $\tilde{u}$ of $u$ such that $\tilde{u}^{*} \Omega_{5}=0$, then we obtain the Lie frame $\tilde{u}: U \rightarrow G$ of type (b-1) with dimension 6 in Theorem 2.1 .

The isotropy subgroup $H_{6}^{2}$ of $H_{5}$ at a point of $W_{6}^{2}$ is given by

$$
H_{6}^{2}=\left\{\tilde{A} \tilde{B} \tilde{C} ; A=\left(\begin{array}{cc} 
\pm 1 & \pm \frac{2}{3} b \\
0 & 1
\end{array}\right), B=\left(b \frac{1}{3}(b)^{2}\right), C=\left(\begin{array}{cc}
0 & -\frac{(b)^{3}}{18} \\
\frac{(b)^{3}}{18} & 0
\end{array}\right)\right\} .
$$

Suppose that there is an open set $U$ in $M$ such that $\left.\lambda\right|_{U}$ is of constant type at sixth order with respect to $W_{6}^{2}$; we say that $\lambda$ is of type (b-2) in $U$. Furthermore we reduce the frames.

Construction of seventh order frames of type (b-2).

The Lie algebra $\mathfrak{h}_{6}$ of $H_{6}^{c}$ is given by 


$$
\mathfrak{h}_{6}=\left\{\left(\begin{array}{ccc}
\alpha & 0 & 0 \\
\beta & 0 & 0 \\
0 & { }^{t} \beta & -{ }^{t} \alpha
\end{array}\right), \alpha=\left(\begin{array}{cc}
0 & \frac{2}{3} \beta_{1} \\
0 & 0
\end{array}\right), \beta=\left(\begin{array}{ll}
\beta_{1} & 0
\end{array}\right)\right\} .
$$

We decompose $\mathfrak{h}_{5}=\mathfrak{h}_{6}+\mathfrak{m}_{6}$, and for a basis of $\mathfrak{m}_{6}$ we take

$$
e_{9}=\left(\begin{array}{rr|r|rr}
1 & 0 & 0 & 0 & 0 \\
0 & 2 & 0 & 0 & 0 \\
\hline 0 & 0 & 0 & 0 & 0 \\
\hline 0 & 0 & 0 & -1 & 0 \\
0 & 0 & 0 & 0 & -2
\end{array}\right) .
$$

The representation $\rho_{6}: H_{6}^{2} \rightarrow G L(9, \boldsymbol{R})$ given by the adjoint action of $H_{6}^{2}$ on $\mathfrak{m}+\mathfrak{m}_{1}+\cdots+\mathfrak{m}_{6}$ with respect to the basis $e_{1}, \cdots, e_{9}$ is

$$
\rho_{6}(P)=\left(\begin{array}{ccccccccc}
* & 0 & * & 0 & * & * & * & 0 & 0 \\
* & \pm 1 & * & 0 & * & * & * & 0 & 0 \\
* & 0 & * & 0 & * & * & * & 0 & 0 \\
* & 0 & * & \pm 1 & * & * & * & 0 & 0 \\
* & 2 b & * & -2 b & * & * & * & 0 & 0 \\
* & \pm \frac{2(b)^{2}}{3} & * & \mp \frac{2(b)^{2}}{3} & * & * & * & 0 & 0 \\
* & \frac{2(b)^{3}}{9} & * & -\frac{2(b)^{3}}{9} & * & * & * & 0 & 0 \\
* & \pm \frac{(b)^{4}}{18} & * & \mp \frac{(b)^{4}}{18} & * & * & * & \pm 1 & 0 \\
* & -b & * & \frac{2 b}{3} & * & * & * & 0 & 1
\end{array}\right) \text {, }
$$

where $P \in H_{6}^{2}$. With respect to $\mathfrak{h}_{5}=\mathfrak{h}_{6}+\mathfrak{m}_{6}$, we have $\Omega_{5}=\Omega_{6}+\Theta_{6}$, where

$$
\Theta_{6}=\omega_{1}^{1} e_{9} \text {. }
$$

We consider orbits and local sections $W_{7}$ of the action of $\left(H_{6}^{c}, \rho_{6}\right)$ on $G_{9,1}$. We can take

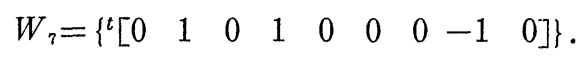

Construction of eighth order frames of type (b-2).

The isotropy subgroup $H_{7}$ of $H_{6}^{c}$ at a point of $W_{7}$ is given by

$$
H_{7}=\left\{\left(\begin{array}{ccc}
A & 0 & 0 \\
0 & I_{2} & 0 \\
0 & 0 & { }^{t} A^{-1}
\end{array}\right) ; A,\left(\begin{array}{cc} 
\pm 1 & 0 \\
0 & 0
\end{array}\right)\right\} \text {. }
$$


The Lie algebra $\mathfrak{h}_{7}$ of $H_{7}$ is equal to 0 . For a basis of $\mathfrak{h}_{6}$ we take

$$
\begin{gathered}
e_{10}=\left(\begin{array}{cc|c|cc}
0 & 1 & 0 & 0 & 0 \\
0 & 0 & 0 & 0 & 0 \\
\hline \frac{3}{2} & 0 & 0 & 0 & 0 \\
\hline 0 & 0 & \frac{3}{2} & 0 & 0 \\
0 & 0 & 0 & -1 & 0
\end{array}\right) \\
\Omega_{6}=\omega_{1}^{2} e_{10} .
\end{gathered}
$$

As the result we take a local cross section

$$
W_{8}=\left\{\left[\begin{array}{llllllllll}
0 & 1 & 0 & 1 & 0 & 0 & 0 & -1 & 0 & \frac{3}{2} k
\end{array}\right]\right\}
$$

of trivial adjoint action $\left(H_{7}, \rho_{7}\right)$ on $G_{10,1}$. The smooth function $k$ on $U$ is called the seventh order invariant of $\left.\lambda\right|_{U}$. The Lie frame of a Legendre map $\lambda: U \rightarrow$ $T_{1} S^{2}$ of type (b-2) is the eighth order frame field $u$ along $\lambda$ with respect to $W_{8}$. In this way we obtain the Lie frame of type (b-2) in Theorem 2.1.

We have thus proved Theorem 2.1. Figure 1 shows the Cartan polygon. (See e.g [Gre].)

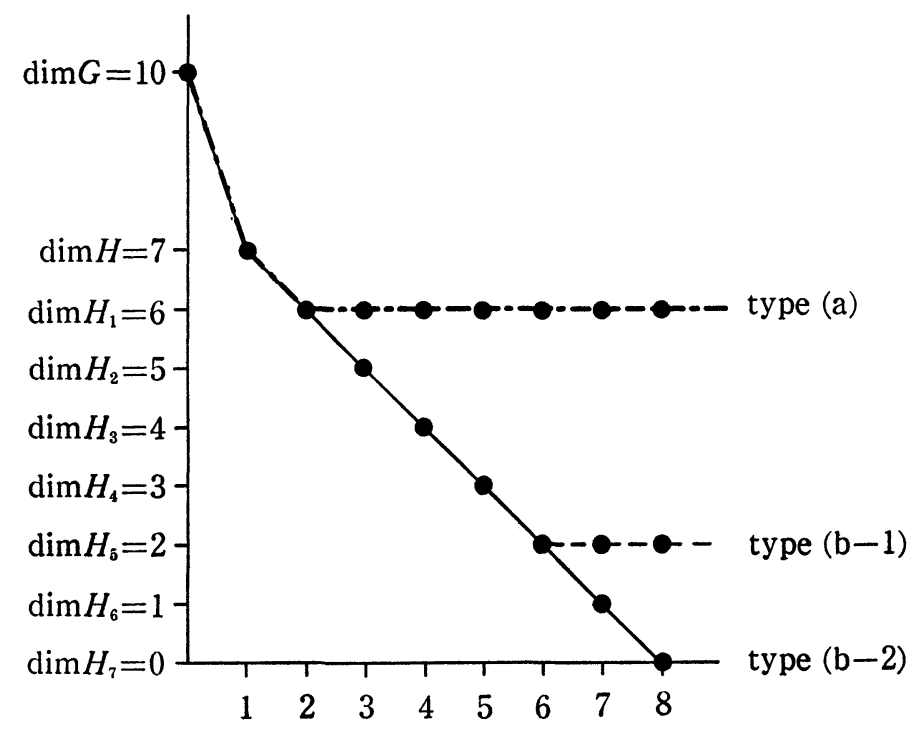

Figure 1 


\section{Classification of Legendre maps in $T_{1} S^{2}$ in view of curvature curves}

Let us consider the classification of curves obtained in Theorem 2.1 in view of curvature circles. The curvature circle of $\lambda: M \rightarrow T_{1} S^{2}$ of type (a), (b-1) or $(\mathrm{b}-2)$ is $[K]=\left[Y_{5}\right]$. We begin considering curves of type (a).

COROllary 4.1. A Legendre map of type (a) is an oriented circle.

Proof. $[K]$ is constant since $d Y_{5}=0$. Thus $\lambda$ is an oriented circle.

For a curve of type (a) we can take the following Lie frame:

$$
\left(Y_{1}(s) Y_{2}(s) Y_{3}(s) Y_{4}(s) Y_{5}(s)\right)=\left(\begin{array}{cc|c|cc}
1 & 0 & s & \frac{s^{2}}{2} & 0 \\
0 & 1 & 0 & 0 & 0 \\
\hline 0 & 0 & 1 & s & 0 \\
\hline 0 & 0 & 0 & 1 & 0 \\
0 & 0 & 0 & 0 & 1
\end{array}\right)=\exp s X_{a},
$$

where $X_{a}$ is the matrix in (2.22). By using the coordinate of $\boldsymbol{R}_{2}^{n+3}$ so that its scalar product $\langle$,$\rangle is given by (1.1), we describe \exp s X(0)$ as follows:

$$
\begin{aligned}
s \rightarrow \exp s X_{a}(o) & =\text { Line }\{(1, \cos t, \sin t, 0,0),(0,0,0,1,1)\} \in \Lambda^{3} \\
& =((\cos t, \sin t, 0),(0,0,1)) \in T_{1} S^{2},
\end{aligned}
$$

where $s=2 \tan t / 2$. This curve is obviously an oriented circle.

Now we consider curves of type (b-1). For a curve of type (b-1) we can take the following Lie frame:

$$
\left(Y_{1}(s) Y_{2}(s) Y_{3}(s) Y_{4}(s) Y_{5}(s)\right)=\left(\begin{array}{cc|c|cc}
1 & 0 & s & \frac{s^{2}}{2} & -\frac{s^{3}}{6} \\
s & 1 & \frac{s^{2}}{2} & \frac{s^{3}}{6} & -\frac{s^{4}}{24} \\
\hline 0 & 0 & 1 & s & -\frac{s^{2}}{2} \\
\hline 0 & 0 & 0 & 1 & -s \\
0 & 0 & 0 & 0 & 1
\end{array}\right)=\exp s X_{b}^{1},
$$

where $X_{b}^{1}$ is the matrix in (2.23). We describe $\exp s X_{b}^{1}(0)$ as follows :

$$
\begin{aligned}
& s \rightarrow \exp s X_{b}^{1}(o)=(f(s), \xi(s)) \in T_{1} S^{2} \\
& f(s)=\frac{1}{\Delta}\left(-s^{6}+36 s^{4}-144 s^{2}+576,12 s^{5}+576 s,-96 s^{3}\right) \\
& \xi(s)=\frac{1}{\Delta}\left(-192 s^{3},-24 s^{4}+288 s^{2},-s^{6}-36 s^{4}+144 s^{2}+576\right),
\end{aligned}
$$


where $\Delta=s^{6}+36 s^{4}+144 s^{2}+576$. Figure 2 and Figure 3 show the curves $f$ and $\xi$ on $S^{2} \subset \boldsymbol{E}^{3}$ respectively.

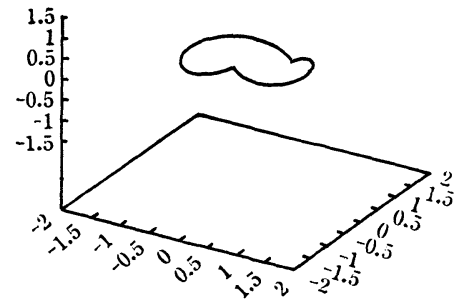

Figure 2

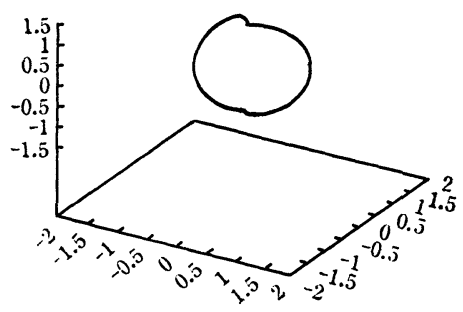

Figure 3

We mention the procedure for distinguishing the type of a given curve by the curvature circle. Let $s \rightarrow \lambda(s)$ be a curve in $T_{1} S^{2}$ and $[K(s)]$ the curvature circle of $\lambda(s)$. When $d K / d s=0, \lambda$ is an oriented circle. If $d K / d s \neq 0$ we examine the second differential $d^{2} K / d s^{2}$. We let $d^{2} K / d s^{2}=k K-Y$. If $k \neq 0$ then $\lambda$ is a general curve with $k \neq 0$. If $k=0$ we examine the fifth differential $d^{5} K / d s^{5}$. When $d^{5} K / d s^{5}=0, \lambda$ is a curve of type (b-1). When $d^{5} K / d s^{5} \neq 0, \lambda$ is a general curve with $k=0$. Figure 4 summarizes the procedure above.

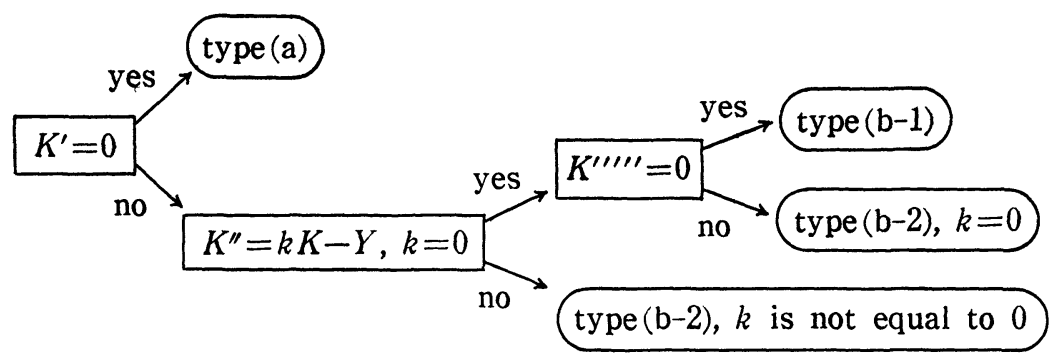

Figure 4

Finally we obtain the necessary and sufficient condition that two curves are Lie equivalent by virtue of Theorem 2.1 and the theorem about uniqueness and existence of mapping of a manifold into a Lie group (see e.g. [Gri §1]).

COROLlARY 4.2. (a) Any two oriented circles in $T_{1} S^{2}$ are Lie equivalent.

(b) Any two curves of type (b-1) in $T_{1} S^{2}$ are Lie equivalent.

(c) Let $\lambda: M \rightarrow T_{1} S^{2}, \tilde{\lambda}: \tilde{M} \rightarrow T_{1} S^{2}$ be smooth curves of type (b-2), and $k, \tilde{k}$ the seventh order invariant of $\lambda, \tilde{\lambda}$ respectively. Curves $\lambda$ and $\tilde{\lambda}$ are Lie equivalent if and only if there exists a one-to-one correspondence $\varphi: M \rightarrow \tilde{M}$ such that $k=\varphi * \tilde{k}$. 


\title{
REFERENCES
}

[C] T.E. CECIL, Lie Sphere Geometry, Springer-Verlag, New York, 1992.

[CC] T.E. Cecil And S.S. Chern, Tautness and Lie sphere geometry, Math. Ann., 278 (1987), 381-399.

[Gre] M.L. GREEN, The moving frame, differential invariants and rigidity theorems for curves in homogeneous spaces, Duke Math. J., 45 (1978), 735-779.

[Gri] P. Griffiths, On Cartan's method of Lie groups and moving frames as applied to uniqueness and existence questions in differential geometry, Duke Math. J., 41 (1974), 775-814.

[J] G. R. JENSEN, Higher Order Contact of Submanifolds of Homogeneous Spaces, Lecture Notes in Math., 610, Springer-Verlag, Berlin, Heidelberg, New York, 1977.

[M] R. MiYaokA, Lie contact structure and normal Cartan connections, Kodai Math. J., 14 (1991), 13-41.

[P] U. Pinkall, Dupin hypersurfaces, Math. Ann., 270 (1985), 427-440.

[SS] S. SASAKI AND T. SUGURI, On the problems of equivalence of plane curves in the Lie's higher circle geometry and of minimal curves in the conformal geometry, Tôhoku Math. J., 47 (1940), 77-86.

[SY] H. Sato and K. Yamaguchi, Lie contact manifolds, Geometry of Manifolds (K. Shiohama, ed.), Academic Press, Boston, 1989, 191-238.

[Y] T. YAMAZAKI, Dupin hypersurfaces and Lie sphere geometry, Master thesis at Tôhoku University (1988), (in Japanese).

[YY] T. YamaZaKi and A.Y. Yoshikawa, Classification of surfaces in three-sphere in Lie sphere geometry, to appear in Nagoya Math. J., 143 (1996).

\author{
SoKahigashi High SchOOL \\ KAKINOKI-CHO 1110 \\ SoKa, SAITAMA 340 \\ JAPAN \\ Graduate School of Polymathematics \\ NAGOYA UNIVERSITY \\ CHIKUSA-KU, NAGOYA 464-01 \\ JAPAN
}

